\title{
SOCIALIZACIÓN INFANTIL Y ESTILOS DE APRENDIZAJE. APORTES PARA LA CONSTRUCCIÓN DE MODELOS DE EDUCACIÓN INTERCULTURAL DESDE LAS PRÁCTICAS COTIDIANAS EN UNA COMUNIDAD CH'OL
}

\author{
Kathia Núñez Patiño \\ kathia.nunez@unach.mx \\ Cecilia Alba Villalobos \\ cecilialba03@hotmail.com \\ Facultad de Ciencias Sociales \\ Universidad Autónoma de Chiapas \\ México
}

\section{RESUMEN}

En este artículo se exponen las principales características de los estilos de aprendizaje expresados en las prácticas cotidianas de una comunidad ch'ol, señalando la importancia de integrar los modelos educativos interculturales y las relaciones que se establecen para el aprendizaje en los contextos domésticos y de la comunidad. Son estos elementos los que pueden permitir una continuidad socializadora de los niños en los espacios áulicos con base en los valores, metas y conocimientos culturales de las localidades donde se implementan los programas educativos oficiales, los cuales hasta el momento, sólo representan los valores e intereses del estado nacional.

Palabras clave: socialización infantil, aprendizaje, educación intercultural. 


\begin{abstract}
This paper presents the main learning style characteristics expressed in the everyday practices of a ch'ol community. It points to the importance of integrating intercultural educational models and learning-oriented relationships in everyday settings and community contexts. It is precisely these elements that can allow children continuity in their socialization process in the classroom based on the cultural values, goals and knowledge of the locations where official educational programs are implemented, which so far only represent the values and interests of the Mexican State.
\end{abstract}

Key words: children's socialization, learning, intercultural education. 


\section{INTRODUCCIÓN}

La educación intercultural se encuentra en un debate abierto con diversas posturas y expresiones, donde se pueden ubicar dos posicionamientos generales en la lid; por un lado, la de mantener el actual sistema de relaciones de dominación en los estados nacionales $\mathrm{y}$, por otro, la de trasformar las relaciones de poder en la lucha por los derechos culturales de los actuales movimientos sociales.

Sin duda, esta falta de respuesta por parte del Estado nacional en México para generar los mecanismos a través de los cuales se puedan crear los espacios reales de participación política, basados en los contextos culturales de los pueblos originarios, manifiesta su crisis actual frente a estas demandas, pero también frente a las exigencias del capitalismo en su fase neoliberal actual.

Esta disyuntiva ha generado formas distintas en las que se piensa y expresa la educación intercultural: mientras que el Estado establece dentro de los programas educativos contenidos interculturales, sólo para indígenas y elaborados desde los espacios de poder en donde se considera la cultura como una lista de características étnicas, las comunidades han avanzado en la construcción de propuestas pedagógicas emanadas desde las luchas y necesidades de los pueblos, teniendo como fuente generadora de contenidos pedagógicos sus prácticas cotidianas; generando al mismo tiempo procesos de recuperación, reproducción, valorización y potencialización cultural, desde una postura crítica frente a la colonización occidental, base del actual sistema de dominación.

Ejemplo de estas experiencias son las escuelas autónomas zapatistas y el modelo curricular de educación primaria intercultural y bilingüe de la Unión de Maestros de la Nueva Educación para México, UNEM. Es en este contexto donde exponemos un esbozo teórico que nos ha posibilitado identificar los principales estilos de aprendizaje infantil que se manifiestan en las relaciones expresadas en las prácticas cotidianas, donde los niños aprenden en su cultura, dentro de una comunidad ch'ol; elementos poco abordados de manera explícita en los diversos posicionamientos para una educación 
intercultural, pero que de una u otra manera se pueden ver reflejados en las propuestas pedagógicas de las escuelas autónomas zapatistas y de UNEM, de ahí la importancia de exponer sus características generales, las cuales han impactado en la construcción de un marco político pertinente para que se reconozcan los estilos culturales propios de aprendizaje de las diversas infancias.

Por ello pensamos pertinente considerar las disposiciones culturales para el aprendizaje que se van construyendo desde el espacio doméstico y de la comunidad, en el caso rural, que es el ámbito que aquí presentamos, pero que también se aplica a los espacios urbanos como el barrio, y que la escuela debería reconocer e integrar dentro de los procesos de aprendizaje formal.

\section{INFANCIA, SOCIALIZACIÓN Y APRENDIZAJE}

Por lo general, los estudios de la infancia se enfocan en tres contextos espaciales: la escuela, la ciudad y la casa, explorados como estructuras que son condicionadas para el control y regulación del cuerpo y la mente del niño a través de regímenes de disciplina, aprendizaje, desarrollo, maduración, frecuentemente considerados como sitios estructurados por los maestros, padres y grupos generacionales.

Los enfoques de la infancia también están muy centrados en contextos urbanos, en este sentido, el artículo se incorpora a los esfuerzos por aportar datos que provengan de espacios rurales, con la finalidad de ofrecer otras ventanas al estudio de otras infancias.

Por ejemplo, en el espacio de la comunidad, que a su vez contiene más espacios, se crean configuraciones que le dan sentido al espacio social desde una visión del mundo particular, a través de las interacciones cotidianas entre los diferentes agentes que participan en la socialización. De tal forma que la socialización más que determinada por las estructuras es producto y condición de ese proceso social.

Además de los espacios sociales, autores como James, Jenks y Prout (1998), señalan dos diferencias que hay que establecer en relación al tiempo infantil, ya que el tiempo 
de la infancia contextualiza la forma y manera precisa en la cual, cualquier particularidad de los niños es habilitada para vivir sus vidas como niños. Por un lado, el tiempo de la infancia, como un período en el curso de la vida que se encaja dentro de la estructura social de cada cultura y, por el otro, el tiempo para los niños, es decir, la experiencia y participación de los niños en los ritmos temporales de la infancia a través de los cuales sus vidas se despliegan.

Esta nueva búsqueda por estudiar al niño como participante activo en su estatus social, político y económico como sujeto contemporáneo, incide en nuevas ideas para abordar el concepto que implica su relación con otros agentes a través de complejos procesos de interacción: la socialización.

\section{LA SOCIALIZACIÓN: EL ROL PARTICIPATIVO DE LOS NIÑOS}

Por socialización, en términos generales, se ha entendido el proceso en el que se trasmiten y configuran los conocimientos, los modos de percibir y categorizar la realidad y los valores socialmente determinados que se encuentran en la base de sustentación de las dimensiones propias del orden social.

La socialización infantil se ha entendido como el proceso a través del cual los niños aprenden una serie de elementos como las creencias, comportamientos y sentimientos de acuerdo con el rol que desempeñan en su cultura, entendiendo rol como la expectativa que se espera del niño según su posición dentro del grupo social. En otras palabras, este proceso «se refiere a las maneras como los niños llegan a ser miembros competentes social y culturalmente al interior del grupo social en que viven» (Terceros 2002: 37).

Estas concepciones de socialización refieren al proceso general; sin embargo, la sociología ha comenzado una crítica del concepto, ya que:

En los estudios sociológicos de la infancia, la socialización se toma a menudo como sinónimo de aculturación, porque este término implica que el niño adquiere la cultura del grupo humano en el 
que se encuentra. Sin embargo, los niños no son vistos como individuos totalmente preparados para participar en el complejo mundo de los adultos, pero comienzan a tener el potencial para ser lentamente introducidos dentro del contacto con las existencias humanas (Ritchie y Collar 1964, en James et al. 1998: 23-24).

De esta manera se critica la socialización entendida como la internalización de la coacción social, proceso que ocurre a través de la regulación de las estructuras, entendidas como externas; en lugar de considerar la socialización como un proceso que surge de las interacciones cotidianas, en una especie de negociación dinámica y continua, en la que se abandona la visión de un niño pasivo y se aborda como uno que participa de manera activa en la socialización y que va emergiendo gradualmente.

Las principales críticas surgen de diferentes disciplinas que rescatan esta visión del niño como agente. Por ejemplo, en la línea de estudios que se ha denominado socializaciónen-la-interacción se toma en cuenta la variedad, la creatividad y preferencias individuales como componentes del proceso socializador (Morton y Wentworth, en De León 2005). Es con estos estudios que los niños empiezan a definirse como actores o agentes que, más que ser objetos preprogramados, son sujetos que se forman a través de actividades interpersonales.

Desde la lingüística también se han desarrollado perspectivas que contribuyen a la discusión sobre la socialización a partir de la adquisición de la lengua, como la perspectiva que proponen Elinor Ochs y Bambi B. Schieffelin (1986: 164-165) ${ }^{1}$, en la que sostienen que la socialización del lenguaje comienza desde el momento del contacto social en la vida de la existencia humana, poniendo énfasis en la socialización como un proceso interactivo. En este sentido, el niño o el novato no es un recipiente vacío del conocimiento sociocultural sino un contribuidor de los significados y resultado de interacciones con otros miembros del grupo social.

Asimismo, autoras como Lourdes de León, también desde los estudios de la socialización lingüística y la adquisición de la lengua, introduce la noción de 
participante porque nos «permite entender la presencia del infante en la interacción y comunicación preverbal antes de la producción lingüística y su capacidad de aprender en estructuras participativas complejas en donde tiene que trabajar identidades fluctuantes de diversos participantes» (2005: 06).

Por otro lado, desde la sicología cultural, Barbara Rogoff aporta elementos en la misma dirección de considerar al niño como participante. La autora se centra en cómo la socialización de los niños se da en su participación en actividades cotidianas con la guía de los adultos, de tal forma que propone el concepto de participación guiada. Este concepto se define como «un proceso en el que los papeles que desempeñan el niño y su cuidador están entrelazados, de tal manera que las interacciones rutinarias entre ellos y la forma en que habitualmente se organiza la actividad proporcionan al niño oportunidades de aprendizaje tanto implícitas como explícitas» (Rogoff 1993: 97). Aquí la interacción y los arreglos o disposiciones entre niños y sus cuidadores son la base de la discusión. Las estrategias de los niños son complementadas en la interacción adultoniño, donde el niño está en una inmersión gradual para su adquisición de las habilidades y creencias propias de su sociedad.

Estas ideas sobre la participación de los niños con otros —adultos o niños más hábiles- que los guían en su socialización, es muy útil, sobre todo si tomamos en cuenta el potencial explicativo que puede resultar a partir de otros estudios en diferentes culturas que aporten más elementos para nutrir el concepto. Sin embargo, como señala Mariëtte De Hann (1999), el concepto de participación guiada puede contener implícitamente la noción de que el aprendizaje de los niños depende del esfuerzo explícito de las instrucciones adultas.

De cualquier forma, la utilidad del concepto de participación guiada es importante porque destaca las diferencias culturales en las que el proceso de socialización se desarrolla al considerar: 
[que] la estructura social y la forma en que tiene la sociedad de organizar la actividad, determinan muchos aspectos del guión y del reparto de papeles, por ejemplo la escolarización formal es obligatoria o al menos existe, si el trabajo de los padres está lejos de casa o es, de alguna otra forma, inaccesible a los niños, si el trabajo del adulto requiere alguna fuerza o conocimiento especializado inasequible a los niños, también hasta qué punto el grado en que se produce una separación de los papeles de niños y niñas, en función de su sexo, y, finalmente, de qué forma se coge a los niños para llevarlos de un sitio a otro (Rogoff 1993: 122).

Las diferentes formas en que los niños participan en la organización de actividades cotidianas que cada sociedad establece, nos da la pauta para conocer las diferencias culturales en las que se produce el aprendizaje de los niños. En este sentido, Antonio Paoli, quien ha estudiado procesos de socialización entre los tseltales, da cuenta de diferentes contextos o ámbitos de sentido en los que se produce aprendizaje. Estos ámbitos de sentido, entendidos como:

\footnotetext{
...la realidad contemplada desde ciertas creencias y ciertas relaciones sociales, desde configuraciones escénicas percibidas a partir de alguna visión del mundo y de procesos sociales específicos. El ámbito de sentido supone una historia y es también una potenciación del entorno material y social. En él se dota a la realidad de sentido, de proyecto y de ritmo intencionales. El valor [...] se aplica necesariamente a ámbitos de sentido y entonces se apela a normas sociales de conducta. Estas normatividades constituyen contextos de legitimidad que regulan a los ámbitos de sentido y presuponen modos de acción y reacción social (Paoli 2003: 15-16).
}

Los ámbitos de sentido, entendidos como la realidad que se configura en espacios físicos como: la comunidad, la casa, la milpa, el monte y el arroyo, ${ }^{2}$ son de utilidad para explorar la forma en que pueden ser explícita o implícitamente estructurados para ofrecer al niño formas de aprendizaje en su socialización, de tal forma que los espacios y tiempos de la infancia pueden estar regulados, pero también se puede escapar de esa regulación, abriendo espacios autónomos de participación infantil.

Con estas aportaciones teóricas, podemos concluir que la socialización infantil es un proceso dinámico en el que los niños aprenden su cultura, como sujetos con existencia propia y agentes que interpretan, reproducen, cuestionan y trasforman la realidad que 
están conociendo, a partir de las interacciones cotidianas que establecen con los demás miembros del grupo del cual son parte. Asimismo consideramos que en la socialización los niños crean espacios autónomos fuera de la mirada adulta recreando su propia realidad. Este proceso puede ser considerado como universal en cuanto que se da en todas las sociedades, pero con características particulares que cada cultura le imprime, dependiendo de esas características culturales, el proceso puede establecer espacios que permitan una mayor participación infantil o la restrinjan.

\section{APRENDIZAJE: DIFERENCIAS CULTURALES QUE DEFINEN LAS FORMAS DE PARTICIPACIÓN INFANTIL}

Estas nuevas perspectivas de la socialización, donde el niño es visto como participante activo, sin duda se remiten a los aportes de la Psicología Rusa, principalmente en los trabajos de Lev S. Vygotsky, y más tarde algunos de sus discípulos, como A. N. Leontyev, retoman sus ideas para generar la teoría de la actividad que centra su interés en la relación entre la conciencia y el mundo material. Para estos autores, el problema surge de una posición teórica en el debate de Marx y Engels sobre la ideología y la crítica del primero a las teorías materialistas.

Esta crítica hacia las teorías materialistas la encuentra Duranti (2000), quien establece que la teoría de la actividad de Vygotsky responde a la pregunta formulada por Marx: ¿cómo elaborar una teoría de la mente humana que se tome en serio el hecho de que los sujetos pensantes no solamente piensan, sino que también se mueven, construyen, tocan, sienten y, sobre todo, interactúan con otros seres humanos y objetos materiales por medio de la actividad física y semiótica?

La respuesta, dice Duranti, trata de reconciliar lo que parecen ser procesos cognitivos individualmente controlados por actuaciones públicas mediante la interacción, donde los individuos participan de forma conjunta en actividades que son más que la mera suma de sus partes. De esta manera, la solución que ofrece la teoría de la actividad es la de invertir la relación habitual entre el individuo y la sociedad, en lugar de pensar desde el individuo y considerar que la actividad social conjunta es la suma de procesos y 
acciones cognitivas individuales, propone una teoría de desarrollo, donde las facultades individuales surgen de procesos interaccionales.

Esta discusión normalmente no se presenta en la psicología cognitiva norteamericana y muchos asumen que también en la teoría de Jean Piaget, psicólogo construccionista. Sin embargo, Barbara Rogoff plantea que:

\begin{abstract}
...es justo decir que tanto Piaget como Vygostky tuvieron en cuenta los procesos sociales y naturales del desarrollo, señalando que, por una parte, Piaget se fijó, sobre todo, en el individuo que ocasionalmente interactúa con otros cuando resuelve problemas lógicos de origen social; por otra parte, Vygotsky prestó especial atención a la participación del niño, junto con otras personas, en el orden social (Rogoff 1993: 64).
\end{abstract}

En cierta forma, Piaget consideraba que el aprendizaje de los niños era más eficaz entre iguales, es decir, con otros niños de igual competencia y no con los adultos por el tipo de relaciones asimétricas que medían entre ellos.

Rogoff trata de conciliar las teorías de estos dos autores, al señalar los intereses particulares de cada uno de ellos, en los que enfatizan: uno en la idea del desarrollo natural y evolutivo y el otro en el contexto de las relaciones sociales del niño en su desarrollo; eligiendo una sobre la otra para fomentar sus propias teorías. La idea, para Rogoff, es rescatar los dos planteamientos como soportes inseparables del desarrollo individual.

Sin embargo, las ideas de Vygostky, más que las de Piaget, son retomadas en las recientes teorías antropológicas que toman la cultura como prácticas y no solo como modelos de pensamiento. Principalmente las teorías de la actividad y la Zona de Desarrollo Próximo (ZDP) son centrales en el estudio de la socialización al ofrecer una manera de analizar las relaciones del individuo con el mundo. 
En el caso de la teoría de la actividad, Vygotsky (1988) propone que el sujeto humano actúa sobre la realidad para adaptarse a ella trasformándola y trasformándose a sí mismo a través de unos instrumentos psicológicos que denomina «mediadores». Este fenómeno, denominado mediación instrumental, es llevado a cabo a través de «herramientas» — mediadores simples, como los recursos materiales - y de «signos» —mediadores más sofisticados, siendo el lenguaje el signo principal—. También establece que la actividad es un conjunto de acciones culturalmente determinadas y contextualizadas que se llevan a cabo en cooperación con otros y la actividad del sujeto en desarrollo es una actividad mediada socialmente.

La actividad que propone Vygotsky es culturalmente determinada y contextualizada, en este sentido, el propio medio humano constituye los mediadores que se emplean en la relación con los objetos, tanto las herramientas como los signos, pero especialmente estos últimos, puesto que el mundo social es esencialmente un mundo formado por procesos simbólicos, entre los que destaca el lenguaje hablado.

El lenguaje, para Vygotsky, es la herramienta que posibilita el cobrar conciencia de uno mismo y el ejercitar el control voluntario de nuestras acciones. Por lo tanto, no se imita simplemente la conducta de lo demás, no se reacciona simplemente al ambiente, sino que con el lenguaje se tiene la posibilidad de afirmar o negar, lo cual indica que el individuo tiene conciencia de lo que es, y que actúa con voluntad propia. En ese momento los individuos llegan a ser distintos y diferentes de los objetos y de los demás, de tal forma que «los sistemas de signos producidos en la cultura en la que viven los niños no son meros "facilitadores" de la actividad psicológica, sino que son sus formadores» (Castorina 1996: 14).

Barbara Rogoff (1993), estudiosa del aprendizaje infantil, es una de las autoras que extiende las ideas de Vygostky, haciendo hincapié no solo en la importancia del contexto cultural para el aprendizaje infantil, sino además en la diversidad de contextos culturales en los que se da la socialización de los niños, argumentando que lo que varía son las formas en el contexto cultural que ofrece diferentes tipos de relaciones, espacios 
y tiempos en el desarrollo de los niños. La autora explora y desarrolla el concepto de ZDP, «de acuerdo con el cual el desarrollo infantil evoluciona a través de la participación del niño en actividades ligeramente distantes de su competencia —en su "zona de desarrollo próximo"-, con la ayuda de adultos o niños más hábiles» (Vygotsky, en Rogoff 1993: 38).

Por otro lado, Mariëtte De Haan, en su estudio del aprendizaje entre los niños mazahuas, va más allá y, si bien reconoce en Vygotsky su formulación sobre las diferencias culturales, plantea que el autor «antepone, en el desarrollo de sus ideas, unas prácticas de enseñanza y unas herramientas de medición a otras, presentándolas como más efectivas y de mayor importancia para el desarrollo» (De Haan 1999: 9).

De esta manera De Haan (1999) defiende la idea de que cada práctica cultural, en principio, crea sus propias formas de aprendizaje y que esas formas de aprender producen el indicio de la práctica cultural de la cual forman parte. Es decir que aprendizaje y cultura son vistos como dos aspectos inseparables del mismo proceso dinámico de desarrollo, donde «las diferencias más importantes entre las culturas ... se relacionan con la variedad de destrezas y valores que definen la meta cultural de la madurez» (Rogoff 1993: 154).

De Hann pone el énfasis en conceptuar las diferencias culturales en las prácticas de aprendizaje. Para esto realiza una revisión de autores que, continuando los aportes de Vygotsky, avanzan en esta dirección, sobre todo desde el concepto de ZDP. Por ejemplo, J. V. Wertsh profundiza sobre la definición de la situación y las metas estructurales de la participación de los niños en relación con los adultos; M. Cole se concentra en el liderazgo de las actividades; B. Rogoff extiende el concepto de ZDP sobre los arreglos implícitos y explícitos en su concepto de Participación Guiada; J. Valsiner también extiende el concepto de Vygotsky, pero desde la constricciónestimulación de la estructura, es decir, retoma las situaciones de conflicto y estimulación del aprendizaje en sus estructuras; Engström, por su parte, se centra en las formas de enseñanza, las cuales desarrollan históricamente nuevas formas de actividad; 
finalmente Leve y Wenger, ponen atención en el aprendizaje situado (en De Hann 1999: 12-15).

Siguiendo las aportaciones de los autores citados, De Haan propone un concepto que ha llamado «creación de situaciones de aprendizaje o de significados que provocan aprendizaje», en el que intenta establecer las relaciones entre el aprendizaje y la cultura, donde las diferencias culturales son reconocidas, tomando en cuenta las múltiples actividades del aprendizaje y una visión en la que el aprendizaje toma espacios como una función en todo tipo de actividades culturales.

Para De Hann, estas nociones pueden dirigir el análisis hacia una visión del aprendizaje que liga de manera efectiva ciertos contextos institucionales con ciertos formatos pedagógicos aprovechados por encima de otros, y posibilitan considerar al aprendizaje basado en prácticas de otras normas culturales y modos de estructurar u organizar las prácticas de aprendizaje. Estos dos aspectos, dice la autora, pueden ser de utilidad para estudiar con mayor profundidad las diferencias culturales en las prácticas de aprendizaje; asimismo, pueden ser considerados como dilemas del aprendizaje, los cuales tienen que ser resueltos en todas las situaciones de aprendizaje y pueden ser resueltos diferentemente en diversas comunidades.

El aspecto sobre las múltiples actividades del aprendizaje tiene que ver con el desarrollo del aprendizaje en el medioambiente o de las disposiciones dentro de las actividades culturales; en lo que respecta a la segunda idea, en la que el aprendizaje toma espacios como una función en todo tipo de actividades culturales, se relaciona con las situaciones o roles, tratando de responder las siguientes cuestiones: ¿cómo, en diferentes culturas, las actividades son trasformadas para aprender o cómo y cuándo son reestructuradas? Y ¿cómo diferentes prácticas culturales toman ventajas de la responsabilidad diferencial o del experto para fomentar aprendizaje? 


\section{SOCIALIZACIÓN, PARTICIPACIÓN E INTERCULTURALIDAD}

La vinculación de aprendizaje y cultura que propone De Hann se complementan con los aportes que ofrece Barbara Rogoff en su concepto de participación guiada, para poder reconocer las diferentes formas en que los niños participan, en interacción con otros, en su socialización. Rogoff retoma el concepto ZDP de Vygotsky, donde el desarrollo del niño es visto como una actividad social que va más allá de su competencia, con la ayuda de adultos. El concepto da cuenta de una región dinámica de la sensibilidad para aprender experiencias en las cuales el desarrollo de los niños es guiado por la interacción social. En esta región es donde cultura y aprendizaje se encuentran (Cole 1981, en Rogoff 1991: 254). En esta zona sensitiva, las variaciones en la interacción social se suponen en la producción de adaptaciones de los individuos en su propio entorno cultural.

Rogoff extiende el concepto de la Zona del Desarrollo Próximo cuando hace hincapié en la interacción de los roles de niños y adultos, en un proceso de participación guiada. La tesis que despliega es que el rápido desarrollo de los niños hacia participantes socializados en sociedad es realizado con una fina combinación de las habilidades de los niños y la guía de los adultos. Rogoff (1991) retoma los aportes de Vygotsky enfatizando el rol del niño como participante activo en su propia socialización. Es decir, que los niños no solo reciben la guía de los adultos, más bien, ellos buscan, estructuran y demandan la asistencia de lo que les rodea para aprender cómo resolver problemas de todo tipo. Refuerza la complementariedad de los roles de los niños y los adultos para fomentar el desarrollo de los niños.

Rogoff (1991) argumenta que la participación guiada de los niños en la interacción social involucra las siguientes actividades:

1) como puente entre habilidades familiares o información que se necesita para resolver un nuevo problema,

2) adaptando y estructurando problema-solución, y 
3) gradualmente trasfiriendo responsabilidades en el manejo de problemasolución para el niño.

Es interesante cómo estas generalizaciones sobre la participación guiada las expone para todo proceso de socialización, donde la articulación de los roles en la socialización puede ser universal, aunque las metas y los significados usados en su implementación varíen en cada cultura. Las variantes están en cómo la interacción de adultos y niños puede ser instrumentada en la socialización.

La idea de participación en el estudio de la conducta humana, de acuerdo con Alessandro Duranti, desde la Antropología lingüística, «significa comprometerse con una investigación detallada y sistemática de los recursos semióticos y materiales que intervienen en la constitución de actividades normalmente conjuntas y con múltiples participantes» (Duranti 2000: 438).

Introducir la participación de los sujetos en la socialización, con una perspectiva contextual como la de Rogoff $(1991,1993)$ en su concepto de participación guiada, y el de las prácticas culturales de Marriëtte De Hann (1999), permiten explorar las formas en que se dan los procesos de estructuración en la socialización, a partir de las prácticas cotidianas en las que se produce el aprendizaje. Esto con la intención de conocer las maneras en que el aprendizaje se estructura cotidianamente en las actividades dentro de la organización social y cómo este se manifiesta o reprime en las prácticas escolares.

Estas aportaciones se dirigen a señalar la importancia de llevar las formas culturales en que los niños aprenden en el espacio de la casa hacia la escuela, con la finalidad de dar continuidad al proceso de socialización infantil. Lo que trae nuevas ideas para la formulación de una educación intercultural en sociedades constituidas por una diversidad cultural, ya que lo intercultural no solo tiene que ver con la inclusión de contenidos relativos a las culturas indígenas, sino también con las formas en que se aprende y actúa en la cultura. 
De esta manera, el acercamiento a las prácticas cotidianas de los niños que aprenden en su cultura ofrece nuevas alternativas en la definición de objetivos para una educación intercultural que pueda vincular conocimientos de la casa con los de la escuela, en función de un desarrollo local, que promueva la participación política de los indígenas en el plano nacional.

\section{PRÁCTICAS Y ESTILOS DE APRENDIZAJE EN UNA COMUNIDAD CH'OL ${ }^{3}$}

Estas nociones son útiles para analizar las múltiples actividades del aprendizaje que se dan entre los niños ch'oles y demás agentes de socialización dentro de la comunidad, en los diferentes ámbitos de sentido donde se producen prácticas de aprendizaje en la socialización infantil, por ejemplo: la casa, la milpa, el juego, el arroyo y la escuela, así como la función de ese aprendizaje en las actividades culturales, con la finalidad de establecer cómo estas actividades son trasformadas implícita o explícitamente para generar aprendizaje.

Se identifica el tipo de participación guiada que se despliega en la socialización de los niños ch'oles de El Bascán, municipio Salto de Agua, Chiapas, para definir cuáles son las formas culturales que de este proceso emanan a partir de su organización sociocultural. Es decir, cuáles son los modelos de persona, los valores y metas culturales que le dan sentido a este proceso de socialización en particular, destacando estas tres dimensiones: el aprendizaje de la formación autónoma de los individuos, la relación entre expertos y aprendices para el aprendizaje, y la diversidad de aprendizajes culturales.

\section{FORMACIÓN AUTÓNOMA DE LOS INDIVIDUOS}

La formación de los niños que se produce en las múltiples interacciones durante las actividades cotidianas de la comunidad se manifiesta principalmente en el respeto que se da a las decisiones de los niños, en tanto son acreedores de las responsabilidades que se generan de ellas. Por ejemplo, si un niño o niña no desea realizar alguna actividad solicitada por sus padres o hermanos mayores, simplemente dice no o se va. Pueden ser 
reprendidos, pero por lo general no se les obliga a realizarla y se opta por un proceso de convencimiento.

Esto no quiere decir que los niños hagan siempre lo que se les da la gana, aquí es donde los contextos culturales, basados en las metas y valores colectivos, se manifiestan en estrategias, más implícitas que explicitas, donde los adultos a menudo hacen comentarios relacionados con la importancia de la participación de todos los miembros de la familia en las múltiples actividades, porque así contarán con lo necesario para vivir. Cuando los niños y niñas manifiestan que saben sembrar y cosechar, recoger leña, hacer tortillas, cazar animales, pescar, cuidar a los más pequeños y alimentar a los animales del corral o cuidar el potrero, son altamente reconocidos.

Otra estrategia es la de contar historias y anécdotas durante la comida, el trabajo y en el tiempo libre, como bañarse en el arroyo; que expresan las consecuencias que las personas han sufrido cuando deciden hacer cosas que van en contra de los valores culturales, de ahí que los niños van teniendo experiencias a partir de las cuales se generan las decisiones acerca de participar o no de las actividades, las cuales no solo afectan a él como individuo, lo que se suele fomentar en contextos occidentales, donde las decisiones de los individuos sólo les afectan a ellos; sino por el contrario, afectan a todos aquellos con los cuales se interacciona en las actividades cotidianas, fomentado la importancia de las relaciones colectivas y su entorno natural.

Este tipo de estrategias de aprendizaje se suele idealizar con simplificaciones de un romanticismo armónico del buen vivir campesino e/o indígena o, por el contario, desdeñar con la finalidad de legitimar las relaciones de los contextos occidentales, donde la actuación del individuo es independiente de los demás y se estimula su independencia. Llegar a estas conclusiones sólo simplifica las implicaciones de contextos culturales basados en metas y valores diferentes, que se manifiestan en los espacios escolares con evidentes desventajas para los niños indígenas, a quienes se les imponen formas diferentes a las propias para el aprendizaje. 


\section{LA RELACIÓN ENTRE EXPERTOS Y APRENDICES}

Las relaciones que comúnmente se observan dentro de los espacios áulicos oficiales tienden a ser verticales, basadas en la autoridad del maestro, en tanto poseedor del conocimiento. Si bien este modelo es ampliamente criticado, sobre todo en lo que respecta a otorgarles el rol pasivo de esta relación a los niños, sigue siendo hegemónico en el sistema educativo, donde toda la estructura institucional de la escuela se impone a los niños indígenas y no dirige su atención, como se pretende señalar aquí a partir de las reflexiones teóricas presentadas, a la importancia de los contextos de aprendizaje infantiles en las comunidades indígenas, para que la escuela sea la que se adapte a la diversidad sociocultural que representan las aulas en México.

Las relaciones que se establecen entre los distintos actores de la socialización son diversas, entre ellas se puede identificar la que los niños cho’les de El Bascán tienen con sus padres, hermanos mayores, familiares y demás personas de la comunidad, basadas en un orden jerárquico de acuerdo con la edad y el género en tanto estos definen las actividades dentro de la organización familiar.

Un análisis profundo de las relaciones de género no es el tema central de este trabajo, pero sí es importante señalar su relevancia y la necesidad de detenerse en él, debido a que es muy común hacer un uso político de generalizaciones sobre el poder que ejercen los hombres sobre las mujeres en los contextos indígenas, con la finalidad de descalificar los valores y costumbres de los pueblos originarios. ${ }^{4}$ Con lo cual, tampoco queremos negar que se dan este tipo de relaciones, como en cualquier otro contexto no indígena.

Lo que interesa señalar es cómo los adultos, en quienes se expresan los rasgos que caracterizan el modelo de persona bajo el cual se socializa a los niños, se encuentran vinculados con la principal actividad productiva en la localidad, que es la agricultura, basada en la siembra de maíz. ${ }^{5}$ Esta tarea es poco valorada para el contexto escolar, incluso podríamos decir que desperdiciada, en tanto que es expresión de múltiples conocimientos y relaciones con el entorno natural y cultural, dado que la milpa es un 
ámbito que le da sentido y estructura a la organización familiar y comunitaria, asignando los roles de sus individuos, donde los hombres son proveedores y las mujeres organizan los recursos de la casa.

Los niños se inician participando en las actividades de manera gradual, siendo la primera infancia la etapa de mayor tolerancia y espacios donde se despliega su agencia y sus cuidadores principales son los hermanos mayores, principalmente las niñas. Es alrededor de los diez años cuando demuestran que saben realizar las actividades cotidianas y asumen la responsabilidad de ellas, momento en el cual la estructura social, basada en el trabajo de la milpa, se impone y el juego termina; pero esta es, al mismo tiempo, producto de un elaborado complejo de metas y valores culturales que la consolidan.

Los adultos son entonces portadores de ese modelo a seguir, que se va trasfiriendo en distintos ámbitos como la milpa, la casa o la comunidad, y mediante estrategias narrativas como las leyendas, sucesos o chismes que portan valores y conductas valoradas o no culturalmente; a partir de las cuales, los niños no solo reproducen, si no que paulatinamente también trasforman por medio de procesos complejos de reelaboración de significados, en los que se incluyen como elementos de modificación la influencia escolar y los medios de comunicación.

Frente a todo este complejo de elementos significativos que los niños indígenas de El Bascán tienen a su alrededor, cobran importancia los modelos educativos interculturales, pues los diversos modelos de persona que se enfrentan no lo hacen bajo relaciones horizontales, sino que compiten bajo una fuerte carga de poder que pone en desventaja al modelo de persona ch'ol, desvalorizada en la escuela y frente a los medios masivos de comunicación.

La relación entre hermanos es altamente significativa para el aprendizaje, pues las relaciones verticales no son tan severas como entre los adultos, sin que esto deje en ventaja a los hermanos mayores sobre los menores; sin embargo, las experiencias que se 
comparten a partir de las actividades cotidianas son fuente de múltiples aprendizajes, lo que nos remite al concepto de participación guiada de Barbara Rogoff, que da cuenta de esa región dinámica de la sensibilidad para aprender experiencias, donde el niño es guiado por la interacción social, espacio donde cultura y aprendizaje se encuentran. Esta relación, que es más de compañeros, no debería dejar de ser considerada dentro de las aulas escolares, pues permitiría, de una manera distinta, que los aprendices pudieran aprovechar la experiencia de los expertos.

\section{LA DIVERSIDAD DE APRENDIZAJES CULTURALES}

Como se ha mencionado, una de las principales actividades es la siembra de maíz; pero no es la única. Dentro de la comunidad de El Bascán, se pueden identificar otras ocupaciones que contribuyen a complementar el ingreso familiar, estas son la cría de ganado y de animales de corral, la de chofer, albañil y profesionistas como la de maestro; además de otras actividades que son características del entorno como la pesca de río y la cacería de animales: tepezcuinte o diversas aves, siendo esta última propia de los niños.

De esta manera la comunidad y sus alrededores ofrecen una gran diversidad de actividades que los niños aprenden cotidianamente y en las que pueden llegar a ser expertos, en una o varias de ellas. En este contexto se manifiesta, de nueva cuenta, esta formación autónoma donde se respetan las preferencias y a partir de éstas se propicia el desarrollo de ciertas habilidades. Es muy común que cuando a los adultos se les pregunta acerca de por qué los niños no realizan ciertas actividades, la respuesta es «no quieren»o «no les gusta hacerlo»; no se les descalifica, como usualmente se hace en el aula escolar, en donde incluso se les señala de tontos.

Esta diversidad de habilidades que los niños pueden desarrollar es un aspecto defendido por diversos enfoques pedagógicos; sin embargo en la práctica escolar se pone mayor énfasis en las matemáticas y la escritura, habilidades altamente valoradas en las sociedades occidentales, que dejan en plena desventaja a los niños indígenas que no logran destacar en estas áreas, siendo objeto de humillaciones. De esta manera, dentro 
de la estructura escolar, la relación entre conocimientos formales que ofrece la escuela y los no formales que los niños indígenas adquieren en su familia y comunidad se ven enfrentados en relaciones de poder que complican los procesos de aprendizaje.

\section{REFLEXIONES FINALES: MODELOS DE EDUCACIÓN INTERCULTURAL}

En el caso de México la discusión en torno a la diversidad que nos caracteriza como país, alcanzó en 1992 un punto relevante al declararse oficialmente la multiculturalidad de nuestra nación. ${ }^{6}$ Este reconocimiento tuvo implicaciones sociales, políticas y particularmente en la educación, pues durante buena parte del siglo XX, en México se planteó como política de Estado la integración de las poblaciones indígenas. Con la idea de conformar la unidad nacional, se buscó la asimilación de las comunidades desde las escuelas, teniendo como punto de partida la castellanización, situación que se viene reproduciendo hasta la actualidad.

Con ello nuestro sistema educativo se convirtió en una de las causas principales de destrucción de las lenguas y culturas indígenas. A pesar de que en 1976 se establece la Dirección General de Educación Indígena, encargada de ofrecer una educación primaria bilingüe bicultural, y de que más tarde, en 1998, se definen los lineamientos para una educación intercultural bilingüe, en las escuelas y en las aulas los maestros indígenas siguen privilegiando el castellano, y las culturas y las lenguas indígenas están prácticamente ausentes de las escuelas destinadas a los niños y niñas de estos pueblos. ${ }^{7}$

Natalio Hernández (2009: 124) reconoce el trabajo y la lucha de los profesionistas indígenas por ser partícipes en el proceso de definición de las políticas educativas dirigidas a sus poblaciones; pero también es crítico de los resultados obtenidos, al considerar que «los avances cualitativos en términos educativos y pedagógicos arrojan resultados muy deficientes y limitados», exponiendo entre las razones de esta situación, la falta de impulso al desarrollo de las lenguas indígenas y a su uso en el ámbito escolar; además de la deficiente profesionalización de los docentes bilingües y un sistema educativo nacional que ve a la educación indígena como inferior. 
Desde su experiencia como profesor bilingüe, Hernández hace un recuento del proceso seguido por algunos de quienes fueron formados en esa política de aculturación, pero que, como él dice, «tomaron conciencia» de su papel «como intermediarios entre el sistema educativo nacional y nuestros propios pueblos, en tanto objetos y sujetos de la educación indígena» (p. 120). Hernández da cuenta de la lucha iniciada en los años setenta con la conformación de organizaciones y consejos y la realización de congresos que reunieron a grupos de profesionistas indígenas, con la finalidad de reflexionar y tomar un posicionamiento acerca de su participación en la constitución de su propio destino como pueblos indígenas:

\footnotetext{
Ha quedado claro para nosotros, que desde la conquista, la educación que se imparte en nuestras escuelas, no es una educación indígena, sino una educación para indígenas, instrumentada para reproducir en cada etapa histórica la dominación cultural ... Se ha esclarecido que es tiempo de ejercer el derecho de buscar la educación que responda a nuestras propias necesidades, a nuestra especificidad cultural ... Nos hemos convencido de que la fuente del conocimiento donde debemos acudir, de donde no debíamos habernos alejado, debe ser la familia, la comunidad indígena, porque en ella está el secreto de la sobrevivencia, está la educación indígena tradicional, de donde tenemos que extraer los conocimientos, para dejar de ser objetos, para convertirnos en sujetos de nuestra propia historia, en constructores de nuestro propio destino. ${ }^{8}$
}

Es decir, si bien para los propios pueblos ha sido clara la necesidad de participar en su proceso educativo, desde su propia cultura, se han enfrentado a una visión de Estado que, a pesar del discurso, en el que reconoce la multiculturalidad, en el fondo y en la práctica apuesta por la homogeneización.

En este sentido es interesante el señalamiento que hacen Elsie Rockwell y Ana María R. Gomes (2009) acerca de que las cosmologías amerindias están desafiando la noción occidental del multiculturalismo a partir del provocativo concepto del «multinaturalismo», noción de que todos los seres naturales son el resultado de la metamorfosis de una humanidad original y universal. Las elaboraciones teóricas latinoamericanas del poscolonialismo ponen el énfasis en los procesos autónomos de decolonización del conocimiento, de sus territorios y recursos naturales y de 
movimientos sociales que también juegan un rol significativo en la reconceptualización de las relaciones en los contextos indígenas hacia la escuela.

El trabajo por una educación indígena diferente tiene como sustento una serie de experiencias comunitarias en distintas regiones del país (Corona y De la Peza 2004) que han profundizado en los contrastes entre la perspectiva teórica de la interculturalidad y su puesta en práctica desde la educación.

Ejemplo de ello son las experiencias autonómicas zapatistas y las de la Unión de Maestros de la Nueva Educación para México, UNEM, en el caso de Chiapas, las cuales nos ofrecen un marco de reflexión para seguir explorando los espacios culturales diversos de los pueblos originarios con los que explicitar, desde las prácticas cotidianas, los conocimientos y relaciones para el aprendizaje, con la finalidad de contribuir a la construcción de modelos interculturales de educación dentro de las demandas políticas de autonomía.

En el caso de las escuelas zapatistas, forman parte del proyecto político de la autonomía indígena de facto, así lo expresan claramente y de manera radical rechazan el modelo intercultural de educación nacional, así como la noción que implica, desde el ámbito oficial, una etapa más de una educación para indígenas, en lo que Rosalva Aída Hernández (2004) denomina neoindigenismo.

Estas escuelas actualmente funcionan en las regiones de influencia zapatista al margen de cualquier tipo de reconocimiento oficial, situación que no les preocupa, pues la educación que se implementa responde a las necesidades de las comunidades indígenas y del propio proceso autonómico zapatista, y no a las de los intereses nacionales, hegemonizados por la elite neoliberal.

Por otra parte, está la propuesta curricular de UNEM, fundada en 1995, que en sus inicios tuvo como antecedente el Proyecto de Educación Comunitaria Indígena, PECI, implementado por la Secretaría de Educación del Estado de Chiapas, SECH, para 
responder a las demandas indígenas del movimiento zapatista. De este proyecto un grupo de «jóvenes educadores comunitarios coincidió en la necesidad de formular una propuesta alternativa de educación de los pueblos y las comunidades tseltales, tsotsiles y ch'oles comisionados por las asambleas de sus comunidades» (UNEM 2009: 34).

Desde su fundación, UNEM ha recorrido un largo camino en el que ha logrado consolidar un posicionamiento político contundente, que se aleja del utópico diálogo intercultural y enfatiza las relaciones de poder asimétricas entre la sociedad nacionalmestiza y las sociedades indígenas, proyectando una educación intercultural, la cual según explican,

...pretende articular los conocimientos indígenas y los occidentales, esta articulación se logra a partir de lo propio, de nuestra vida como indígenas y campesinos, de la que nunca se nos enseñó nada en la escuela, que no aparece en los libros de texto oficiales y que la Secretaría de Educación Pública y los maestros oficiales, mestizos y bilingües, nunca consideraron digna de tomarse en cuenta en la educación escolarizada (UNEM 2009: 58).

Consideramos que, además de su posicionamiento político, que pretende avanzar hacia la construcción de una ciudadanía que integre la diversidad cultural, otro gran avance que el proyecto de UNEM ha desarrollado es su propuesta metodológica para la articulación de los conocimientos indígenas y occidentales desde lo propio, herramienta útil para desarrollar el marco teórico expuesto.

El método que proponen lo denominan inductivo intercultural porque parten de las actividades de la comunidad, bajo la idea de que los niños se integren a la actividad de cualquier tipo, ya sea productiva, social o de fiesta, desde cuatro fases que integran su modelo pedagógico: investigar, explicitar, ampliar el conocimiento, y articular. Esta última fase, la llaman articulación crítica porque resaltan los valores positivos de las sociedades indígenas, como la propiedad comunal y la relación con la tierra, que son contrastados con los conocimientos y valores «universales». De esta manera, su 
propuesta se encamina a la construcción de ciudadanía, exigiendo una educación intercultural como derecho. 


\section{BIBLIOGRAFÍA}

Allison, James, Chris Jenks y Alan Prout, 1998, Theorizing Childhood, Teachers College Columbia University, New York.

Castorina, José Antonio, Emilia Ferreiro, Marta Kohl de Oliveira y Delia Lerner, 1996, Piaget-Vygotsky: contribuciones para replantear el debate, Paidós, México.

Corona B., Sarah y Carmen de la Peza (coords.), 2004, Educación indígena. En torno a la interculturalidad, Universidad de Guadalajara, Universidad Autónoma Metropolitana Xochimilco, México.

De Haan, Mariëtte, 1999, Learning as Cultural Practice. How Children Learn in a Mazahua Community, Netherlands School for Social and Economic Policy Reserch, Amsterdam.

De León, Pasquel, Lourdes, 2005, La llegada del alma: lenguaje, infancia y socialización entre los tsotsiles de Zinacantán, CIESAS, México.

Duranti, Alessandro, 2000, Antropología Lingüística, Cambridge University Press, Madrid.

Hernández Castillo, Rosalva Aída, Sierra y Paz (coords.), 2004, El estado y los indígenas en los tiempos del Pan: neoindigenismo, legalidad e identidad, CIESAS, Porrúa, México.

Hernández, Natalio, 2009, De la exclusión al diálogo intercultural con los pueblos indígenas, Plaza y Valdés, UNICH, México.

Ochs, Elinor y Bambi B. Schieffelin, 1986, «Language Socialization», Annual Review of Anthropology, v. 15, California. 
Paoli, Antonio, 2003, Educación, autonomía y lekil kuxlejal: Aproximaciones sociolingüísticas a la sabiduría de los tzeltales, UAM, México.

Rockwell, Elsie y Gomes, Ana Maria R., 2009, «Introduction to the Special Issue: Rethinking Indigenous Education from a Latin American Perspective», Anthropology \& Education Quarterly, v. 40, i. 2, pp. 97-109.

Rogoff, Barbara, 1991, «The Joint Socialization of Development by Young Children and Adults», en Social Influences and Socialization in Infancy, Edited by Lewis, Michel and Saul Feinman, Plenum Press, New York, London.

1993, Aprendices del pensamiento. El desarrollo cognitivo en el contexto social, Paidós, Barcelona.

Sierra, María Teresa, 2009, «Las mujeres indígenas ante la justicia comunitaria. Perspectivas desde la interculturalidad y los derechos», Desacatos, n. 31, septiembrediciembre, pp. 73-88.

Terceros Ferrufino, Carmen, 2002, «Concepciones de infancia y prácticas comunicativas de socialización en la niñez quechua de Cororo, Bolivia», Tesis de maestría en Lingüística Indoamericana, CIESAS, INI, México.

UNEM, 2009, Modelo Curricular de educación intercultural bilingüe, CIESAS, México.

Vygotsky, Lev S., 1988, Pensamiento y lenguaje, Ediciones Quinto Sol, México. 


\section{Notas}

${ }^{1}$ La traducción de los textos es de las autoras.

${ }^{2}$ Los ámbitos de sentido los entendemos como construcciones abstractas de la realidad y no como espacios físicos.

${ }^{3}$ La mayor parte de la información obtenida en la etnografía es de la comunidad El Bascán, elaborada durante diferentes periodos que iniciaron con el trabajo de campo realizado en el programa de Maestría del CIESAS Sureste en el año 2004 y ha continuado durante dos visitas al año a esta comunidad. Las referencias de las escuelas zapatistas se basan en la etnografía realizada durante el trabajo de Maestría señalado en la región ch'ol, el cual se complementa con acercamientos que se han tenido en este año en la región de Los Altos. De este trabajo etnográfico, resultan las reflexiones entre las autoras que aquí sistematizamos y presentamos.

${ }^{4}$ Un análisis importante en este sentido se puede encontrar en Sierra, María Teresa (2009) «Las mujeres indígenas ante la justicia comunitaria. Perspectivas desde la interculturalidad y los derechos», en Desacatos, n. 31, septiembre-diciembre 2009, pp. 73-88.

${ }^{5}$ Cabe destacar que, si bien la base de la actividad productiva es la siembra del maíz, los pobladores de El Bascán se dedican a otras actividades para complementar sus ingresos como: la cría de ganado extensiva, contratándose como albañiles o choferes e incluso varios tienen profesiones como la de profesor.

${ }^{6}$ La Constitución Política de los Estados Unidos Mexicanos en el artículo $2^{\circ}$ dice: «La nación tiene una composición pluricultural sustentada originalmente en sus pueblos indígenas que son aquellos que descienden de poblaciones que habitaban en el territorio actual del país al iniciarse la colonización y que conservan sus propias instituciones sociales, económicas, culturales y políticas, o parte de ellas».

${ }^{7}$ Conferencia presentada en el Primer Encuentro Interuniversitario de Educación Intercultural, celebrado en la Casa Central de la Universidad de Chile, en Santiago de Chile, los días 10 y 11 de abril de 2008.

${ }^{8}$ Parte de las «Conclusiones del Primer Seminario Nacional de Educación Bilingüe-Bicultural» (mimeo), realizado en la ciudad de Cuernavaca, Morelos, México, en 1979.

Fecha de recepción: 30 de julio de 2010.

Fecha de aceptación: 22 de diciembre de 2010. 